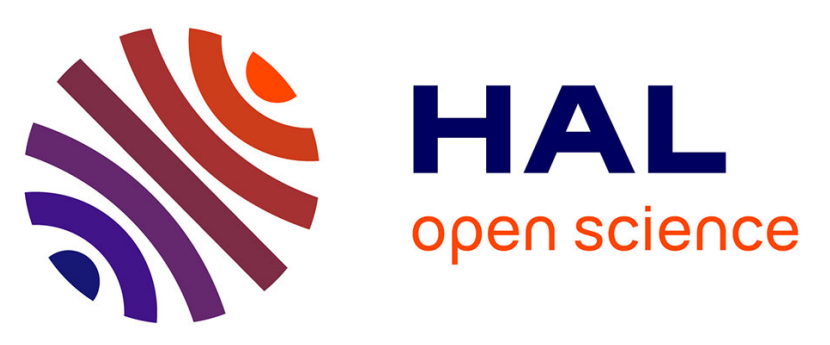

\title{
Hollow Cobalt Phosphide with N-Doped Carbon Skeleton as Bifunctional Electrocatalyst for Overall Water Splitting
}

Yanyu Xie, Minqi Chen, Mengke Cai, Jun Teng, Huanfeng Huang, Yanan Fan, Mihail Barboiu, Dawei Wang, Cheng-Yong Su

\section{To cite this version:}

Yanyu Xie, Minqi Chen, Mengke Cai, Jun Teng, Huanfeng Huang, et al.. Hollow Cobalt Phosphide with N-Doped Carbon Skeleton as Bifunctional Electrocatalyst for Overall Water Splitting. Inorganic Chemistry, 2019, 58 (21), pp.14652-14659. 10.1021/acs.inorgchem.9b02333 . hal-03031011

\section{HAL Id: hal-03031011 \\ https://hal.umontpellier.fr/hal-03031011}

Submitted on 30 Nov 2020

HAL is a multi-disciplinary open access archive for the deposit and dissemination of scientific research documents, whether they are published or not. The documents may come from teaching and research institutions in France or abroad, or from public or private research centers.
L'archive ouverte pluridisciplinaire HAL, est destinée au dépôt et à la diffusion de documents scientifiques de niveau recherche, publiés ou non, émanant des établissements d'enseignement et de recherche français ou étrangers, des laboratoires publics ou privés. 


\title{
Hollow Cobalt Phosphide with N-Doped Carbon Skeleton as Bifunctional Electrocatalyst for Overall Water Splitting
}

\author{
Yanyu Xie, † Minqi Chen, † Mengke Cai, † Jun Teng, † Huanfeng Huang, † Yanan Fan, † Mihail \\ Barboiu,,$+ \ddagger$ Dawei Wang, ${ }^{*},+$ and Cheng-Yong Su† \\ †MOE Laboratory of Bioinorganic and Synthetic Chemistry, Lehn Institute of Functional Materials, \\ School of Chemistry, Sun Yat-Sen University, Guangzhou 510275, China \\ ¥Institut Europeen des Membranes, Adaptive Supramolecular Nanosystems Group, University of \\ Montpellier, ENSCM-CNRS, PI. E. Bataillon CC047, Montpellier 34095, France
}

\begin{abstract}
The development of cost-effective, high-per-formance, and robust bifunctional electrocatalysts for overall water splitting remains highly desirable yet quite challenging. Here, by selecting appreciate precursors of dopamine and a Co-containing metal-organic framework of ZIF-67, we subtly couple their reaction processes to develop a facile approach for the synthesis of a hollow CoP nanostructure with $\mathrm{N}$-doped carbon skeleton (H-CoP@NC). Benefiting from the highly porous nanostructure and conductive carbon skeleton, H-CoP@NC is capable of working as highly active and durable bifunctional electrocatalyst for both hydrogen and oxygen evolution reaction. When further used as the electrocatalyst for overall water splitting, H-CoP@NC delivers excellent activity cell voltage of $1.72 \mathrm{~V}$ at a current density of $10 \mathrm{~mA} \mathrm{~cm}-2$ ), close to that of the noble-metal-based benchmark catalyst couple of $\mathrm{Pt} / \mathrm{C}|| \mathrm{RuO} 2$. Our work thus provides new insights into the development of transitional metal phosphides based hollow hybrid nanostructures, particularly those with multiple functionalities in sustainable energy conversion technologies and systems.
\end{abstract}

\section{Introduction}

The development of clean and recyclable energy source has been urgently required to address the worldwide concerns of environmental pollution and fossil fuel depletion. As a green and renewable energy source, $\mathrm{H} 2$ has been extensively investigated in recent years, as it can be directly generated from water through electrolysis.1,2 The key issue in water electrolysis is to develop highly active, costeffective catalysts for its two half reactions, namely, hydrogen evolution reaction (HER) and oxygen evolution reaction (OER), so as to achieve applicable overall water splitting (OWS) with high current density at low overpotential. The currently commercially available catalysts for HER and OER are Pt/C and ruthenium/iridium oxides, respectively, which possess excellent catalytic activities but are not appropriate for large-scale applications due to their scarcity and high cost. Therefore, alternative materials like the first row transitional metal nitrides (TMNs), oxides (TMOs), phosphides (TMPs), and sulfides (TMSs) have been investigated for HER and OER.3-9 Among these materials, transitional metal phosphides, e.g., Ni2P,10 NiP,11 CoP,12 MoP,13 and so on, are of particular interest due to their bifunctional properties, i.e., electrocatalytic activities for both HER and OER.14-17 Nevertheless, due to the intrinsically low electrical conductivity of pure TMPs, the development of high-performance bifunctional electrocatalysts based on pure TMPs has been hindered.

To improve the electrocatalytic performances of bifunctional TMP catalysts, a general method is to enhance the overall conductivity by combining TMPs with carbonaceous materials like carbon cloth,18 carbon nanotubes,19 graphene quantum dots,20 and reduced graphene oxide.21 The conductive carbonaceous materials also bring additional benefit to the durability of bifunctional TMP catalysts, by 
acting as the supporting skeleton/matrix to stabilize the TMPs and thus enhance their stability. 22 Another approach to the performance improvement of bifunctional TMP catalysts is to design appropriate porous nanostructures that facilitate the efficient exposure of abundant surface active sites for the electro-catalytic HER and/or OER.23-26 Among the various nanostructures that have been developed recently, hollow nanostructures have been of particular interest due to their great structural advantages including low density, large surface area, and shortened paths for mass/charge transport.23,25,27-29 Therefore, by organizing the TMPs and carbonaceous materials into hollow nanostructures, that is, constructing hollow conductive hybrid nanostructures of TMPs and C (denoted as TMPs/C), the overall electrocatalytic perform-ances of TMPs/C for OER and/or HER can be further enhanced.30,31 Despite recent progress, the controlled synthesis of hollow TMPs/C bifunctional catalysts with high electro-catalytic performances remains challenging.

Here, we demonstrate a new approach for the synthesis of a hollow, N-doped carbon-coated CoP nanostructure (denoted as H-CoP@NC) that can be used as highly active bifunctional electrocatalyst for HER and OER (Scheme 1). By selecting appreciate precursors, namely, dopamine and a Cocontaining metal-organic framework (MOF) of ZIF-67,32,33 and subtly coupling their reaction processes, the facile synthesis of H-CoP@NC can be achieved. At slightly basic $\mathrm{pH}$, the decomposition of ZIF-67 into Co-layered double hydroxide (Co-LDH) nanosheets is accompanied by the polymerization of dopamine into polydopamine (PDA) coating, which simultaneously acts as the template for the CoLDH nanosheets to assemble into hollow nanostructures of PDA-coated Co-LDH (H-Co-LDH@PDA). Following such a coupled PDA coating and MOF conversion process, the H-Co-LDH@PDA is further converted into H-CoP@NC through phosphidation calcination. Benefiting from the large inner void and porous thin shell consisting of small CoP nanoparticles and conductive $\mathrm{N}$-doped carbon coating, the $\mathrm{H}$ CoP@NC is able to expose abundant surface active sites and show enhanced electrical conductivity and stability. Therefore, H-CoP@NC delivers great activity and durability when used as bifunctional electrocatalysts for HER, OER, and OWS.

\section{Results and Discussion}

The synthetic procedure for H-CoP@NC is illustrated in Scheme 1. ZIF-67 nanocrystals, which consist of cobalt(II) ions and 2-methylimidazolate ligands, were synthesized and used as the template and cobalt source.34-37 As shown in the scanning electron microscopy (SEM) and transmission electron microscopy (TEM) images (Figure 1a,b), the as-synthesized ZIF-67 are uniform rhombic dodecahedrons with average diameter of about $800 \mathrm{~nm}$. The experimental powder X-ray diffraction (PXRD) pattern of ZIF-67 nanocrystals agrees well with the simulated one,38,39 thus indicating the phase pure nature of ZIF-67 nanocrystal (Figure S1).

Scheme 1. Schematic Illustration of the Synthesis of H-CoP@NC

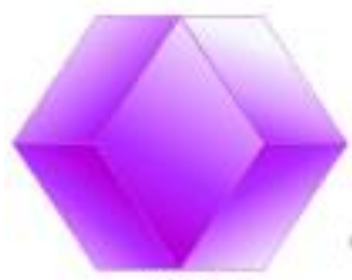

ZIF-67

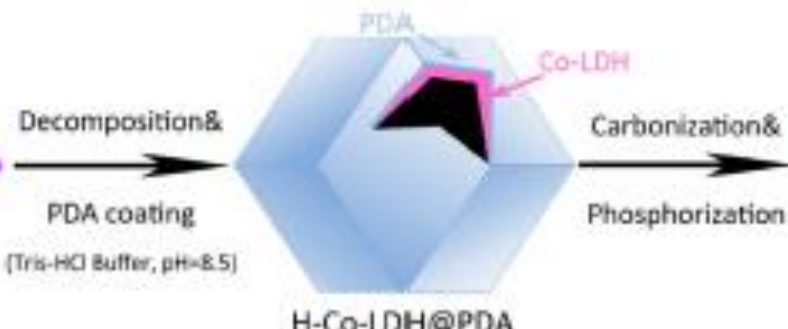

H-CO-LDH@PDA

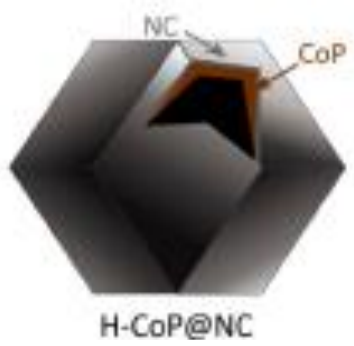

$\mathrm{H}-\mathrm{COP} @ \mathrm{NC}$

The ZIF-67 nanocrystals were then added to a solution containing dopamine hydrochloride and Tris$\mathrm{HCl}$ buffer ( $\mathrm{pH}$ 8.5; see details in the Supporting Information) to allow the coupled PDA coating and MOF conversion processes to occur. In such slightly basic solution, the dopamine monomers are oxidized by the oxygen in ambient atmosphere and spontaneously self-polymerizes into PDA coating 
on ZIF-67 nanocrystals, 40,41 leading to the formation of surface wrinkles in the product (Figures $1 \mathrm{c}, \mathrm{d}$ and S2). The PDA coating can be further verified by the high-resolution TEM (HRTEM) image Figure (e-g)), in which an amorphous layer of ca. $5 \mathrm{~nm}$ in thickness can be found in the product. The formation of PDA coating can be further confirmed by the following experimental results: (1) The Raman spectrum of the product shows no characteristics peaks of their ZIF-67 precursors (Figure S3; 175 and $257 \mathrm{~cm}-1$ for the stretching of $\mathrm{Co}-\mathrm{N}, 682 \mathrm{~cm}-1$ for imidazole ring puckering and $\mathrm{C}-\mathrm{H}$ out of plane bend, $1140 \mathrm{~cm}-1$ for the stretching of $\mathrm{C}-\mathrm{N}$ ), 42,43 which indicates the complete hydrolysis of ZIF67 and the complete removal of the $\mathrm{N}$-containing organic ligands of 2-methylimidazolate. (2) The energy-dispersive spectroscopy (EDX) line scans clearly verify the existence of $N$ and C (Figure $1 \mathrm{~h}, \mathrm{i}$ ), which thus can only be attributed to the $\mathrm{N}$ and $\mathrm{C}$ atoms of PDA layers rather than the 2methylimidazolate ligands. Accompanied by the formation of PDA coating, the decomposition of ZIF67 into Co-LDH nanosheets is simultaneously induced in the slight basic buffer solution, 44 as confirmed by the PXRD patterns (Figures S4 and S5), selected-area electron diffraction (SAED) pattern (Figure S6), and EDX line scans showing the distribution of $\mathrm{Co}$ and $\mathrm{O}$ elements (Figure 1i).35,44-47 TEM image also reveals that Co-LDH possesses a hollow nanostructure (H-Co-LDH), with thin shells of ca. $55 \mathrm{~nm}$ (Figure 1d). Careful examination of a typical H-Co-LDH particle under HRTEM indicates that the shell consists of a large amount of small nanoparticles with average diameter of ca. $12 \mathrm{~nm}$ (Figure 1e). The lattice spacing of 0.274 and $0.164 \mathrm{~nm}$ in these small nanoparticles can be assigned to the (012) and (110) planes of Co-LDH, 45 respectively, thus further confirming that the small nano-particles are Co-LDH (Figure 1(f, g)). Moreover, the distribution of $\mathrm{Co}$ and $\mathrm{O}$ is in line with that of $\mathrm{N}$ and $\mathrm{C}$ in the EDX line scans of $\mathrm{H}-\mathrm{Co}-\mathrm{LDH}$, suggesting the formation of $\mathrm{H}-\mathrm{Co}-\mathrm{LDH}$ particles with homogeneous PDA coating (denoted as H-Co-LDH@PDA). It is worth noting here that although PDA and MOFs have been used together to synthesize hollow PDA nanocapsules 40 or porous hybrid nanostructures, $48-50$ a synthetic approach based on the subtle coupling of PDA coating and MOF conversion processes has not been reported before. Our synthetic approach reported here thus provides new insights into the development of PDA based complex nanostructures.

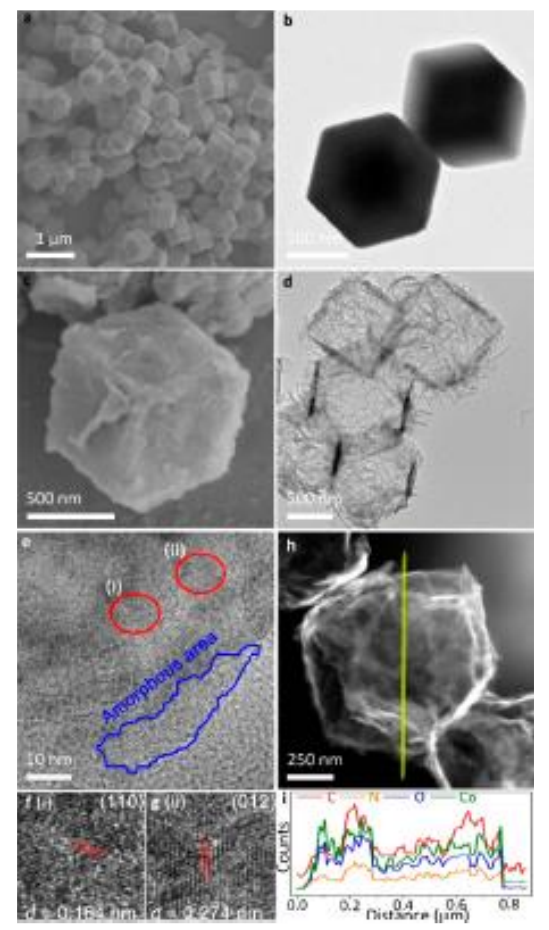

Figure 1. (a) SEM and (b) TEM images of ZIF-67 nanocrystals. (c) SEM, (d) TEM and (e-g) HRTEM images of H-Co-LDH@PDA. (h,i) EDS along a H-Co-LDH@PDA particle. 
We emphasize here that the PDA layer plays a crucial role in the formation of $\mathrm{H}-\mathrm{Co}-\mathrm{LDH}$, by acting as the template to direct the assembly of Co-LDH nanosheets into hollow nanostructure (H-Co-LDH).51,52 In our control experiment without dopamine, the decomposition of ZIF-67 nanocrystal into Co-LDH can still be induced in the slightly basic buffer solution, as confirmed by the PXRD pattern in Figure S7. However, due to the absence of PDA layers, the templated assembly of Co-LDH into hollow nanostructures cannot be achieved, and hollow nanostructures thus cannot be found in the product. Instead, the Co-LDH self-assemblies into nanosheets that are much thicker than those obtained in the presence of dopamine (Figure S8). The notable difference between the nanostructures of the products obtain with and without dopamine thus clearly demonstrates the critical role of PDA layers.

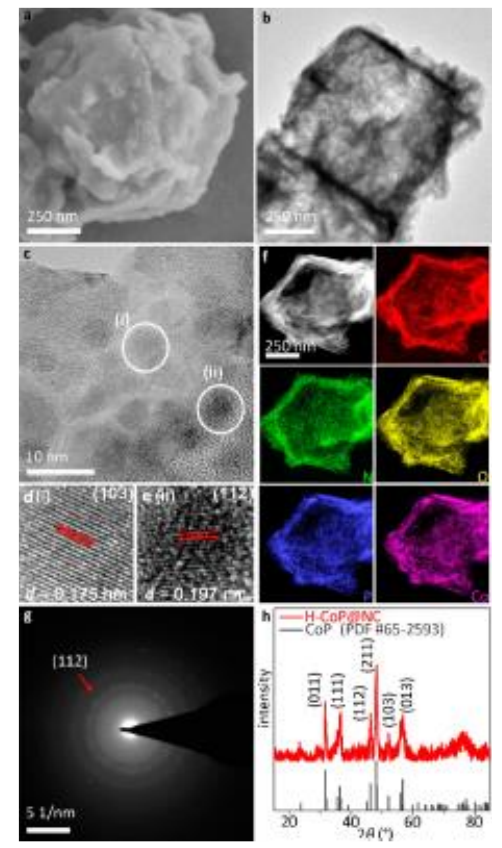

Figure 2. (a) SEM image, (b) TEM image, (c-e) HRTEM images, (f) EDS elemental maps, (g) SAED pattern, and (h) PXRD pattern of H-CoP@NC.

The H-Co-LDH@PDA samples were then subject to pyrolysis at $400{ }^{\circ} \mathrm{C}$ under argon atmosphere and in the presence of sodium hypophosphite (phosphor source), during which $\mathrm{H}-\mathrm{Co}-\mathrm{LDH}$ products were phosphorized and the PDA was carbonized. SEM and TEM images show that as compared with H-CoLDH@PDA the pyrolysis product is further wrinkled, while the hollow nanostructure is retained (Figure $2 a, b)$. Moreover, a large number of small nanoparticles are found to disperse throughout the product. HRTEM image reveals that the small nanoparticles, with an average diameter of ca. $5 \mathrm{~nm}$, can be assigned to orthorhombic CoP, as their interplane distance of 0.175 and $0.195 \mathrm{~nm}$ matches well with that of the (103) and (112) planes of orthorhombic CoP, respectively (Figure 2c-e).53,54 EDX elemental maps of a product particle approve the existence of $\mathrm{Co}$ and $\mathrm{P}$ elements in CoP (Figures $2 \mathrm{f}$ and S9). The EDX elemental maps also show $\mathrm{C}$ and $\mathrm{N}$ elements, which arise from the $\mathrm{N}$-doped carbon coating in the product formed by the carbonization of PDA layer. Moreover, the appearance of $\mathrm{O}$ in the product, as confirmed by the X-ray photoelectron spectroscopy (XPS; cf. the discussion in the following paragraph), is due to the oxidation of superficial CoP by the $\mathrm{O} 2$ in the atmosphere.19,55 The orthorhombic CoP nature of the product particles is further verified by the results from SAED (Figure $2 \mathrm{~g}$ ) and the PXRD pattern (Figure 2h).54,56 Therefore, all these results collectively indicate that the pyrolysis product is hollow nanostructure of $\mathrm{N}$-doped carbon supported CoP nano-particles (H-CoP@NC).

Figure 3a shows the full XPS spectrum of H-CoP@NC, which conforms their composition of Co, P, C, N, and $O$ elements and agrees well with the EDX results (Figures $2 f$ and $S 9$ ). The peaks of Co $2 p 3 / 2$ at 
$778.4 \mathrm{eV}$ and $\mathrm{P} 2 \mathrm{p} 3 / 2$ at $129.3 \mathrm{eV}$ in the high-resolution Co $2 \mathrm{p}$ and $\mathrm{P} 2 \mathrm{p}$ spectra (Figure $3 \mathrm{~b}, \mathrm{c}$ ), respectively, further confirms the existence of CoP in H-CoP@ NC.57 The positive shift of Co 2p3/2 from the binding energies of metallic Co $(778.1-778.2 \mathrm{eV})$ and the negative shift of $2 \mathrm{p} 3 / 2$ from elemental $\mathrm{P}(130.2 \mathrm{eV})$ indicate a partially positive charge of Co atom and a partially negative charge of $\mathrm{P}$ in CoP respectively, 58 which should promote the reactant adsorption and product desorption on the CoP surface, and thus facilitate the electrocatalysis process.11 The existence of $\mathrm{N}$-doped carbon skeleton in H-CoP@NC is evidenced by the high-resolution N 1s spectrum (Figure S10), in which the resolved peaks centered at 398.4, 399.8, and $400.7 \mathrm{eV}$, respectively, can be assigned to the pyridinic $(\mathrm{N}-6)$, pyrrolic $(\mathrm{N}-5)$, and graphitic ( $\mathrm{N}-\mathrm{Q}) \mathrm{N}$ atoms that bond with the $\mathrm{C}$ atoms in the skeleton. $38,59 \mathrm{By}$ calculating the integrated area ratio of these fitted peaks, the proportion of pyridinic, pyrrolic, and graphitic $\mathrm{N}$ atoms is quantified to be $6.1 \%, 40.8 \%$, and $53.1 \%$, respectively. The H-CoP@NC is also analyzed with Raman spectrum (Figure $3 \mathrm{~d}$ ). The $\mathrm{G}$ band $(1354 \mathrm{~cm}-1)$ and $\mathrm{D}$ band $(1588 \mathrm{~cm}-1)$ correspond to graphitic carbon and disordered carbon, respectively, and thus further confirm the formation of carbon skeleton after the pyrolysis at $400{ }^{\circ} \mathrm{C}$. Moreover, based on the full XPS spectrum in Figure 3a, the content of CoP and N-doped carbon is calculated to be 51.3 and $22.6 \mathrm{wt} \%$, respectively. The full XPS spectrum of H-CoP@NC also shows a high content of oxygen (26.1 wt \%), which is evidenced by the by the Co-O bond at $781.7 \mathrm{eV}$ and $\mathrm{P}-\mathrm{O}$ bond at $134.0 \mathrm{eV}$ in the highresolution Co $2 p$ and $\mathrm{P} 2 \mathrm{p}$ spectra in Figure $3 b, c$, respectively, can be attributed to the oxidation of superficial CoP by the 02 in the atmosphere.19,55 Meanwhile, H-CoP@NC is also analyzed by inductively coupled plasma (ICP) spectroscopy and elemental analysis, which gives a much lower content of $\operatorname{CoP}(23.9 \mathrm{wt} \%)$ and much higher content of $\mathrm{N}$-doped carbon (32.5 wt \%). The big differences between the results from XPS and ICP clearly indicate that the CoP nanoparticles tend to distribute within the surface layer of $\mathrm{H}-\mathrm{COP} @ N C$, which should be beneficial to the exposure of more surface active sites for HER, OER and OWS and thus leads to enhanced catalytic activities. The results from XPS and ICP, however, also suggest high content of conductive carbon in the skeleton, which should be a great benefit to the improvement of the overall conductivity and stability of H-CoP@NC and thus could further lead to substantial enhancement in its electrocatalytic activity and durability.
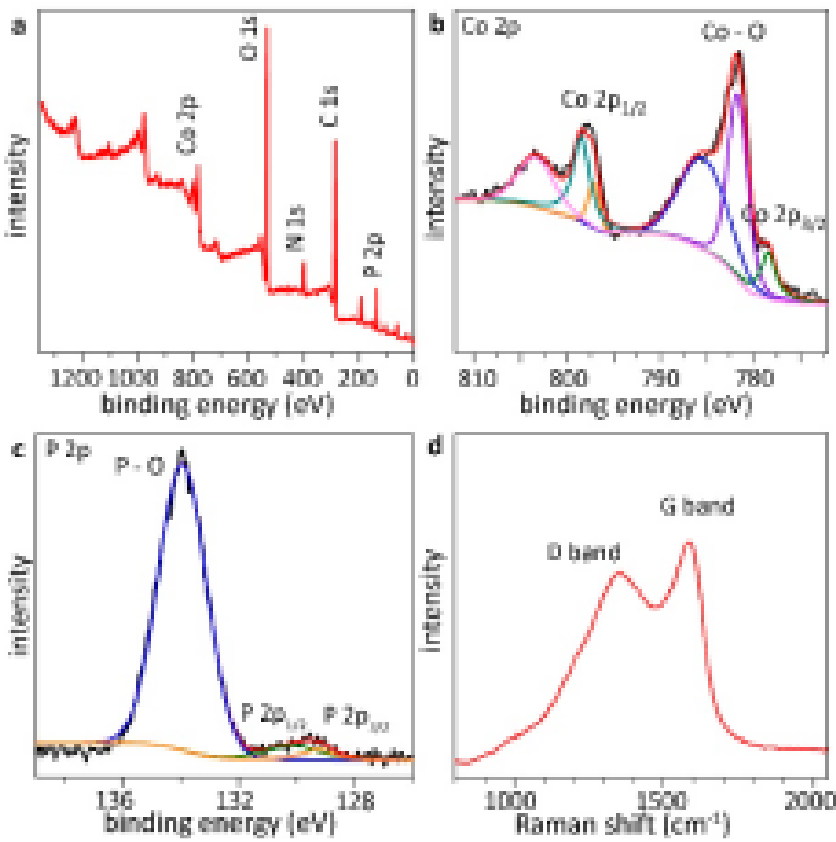

Figure 3. (a) Full XPS spectrum of H-CoP@NC. (b, c) High-resolution XPS spectra of (b) Co 2p and (c) P 2p. (d) Raman spectrum of H-CoP@NC. 
The H-CoP@NC sample was then coated on glassy carbon electrode (GCEs) and its electrocatalytic activities toward OER and HER were evaluated with a typical three-electrode setup (see details in the Experimental Section in the Supporting Information). For comparison, two additional control samples were also synthesized and evaluated, that is, hollow CoP (H-CoP; without carbon support) synthesized by phosphorizing hollow Co-LDH (Figures S11-S16) and CoP/NC synthesized by phosphorizing ZIF-67 without PDA coating (Figures S17 and S18). The electrocatalytic activities of these catalysts were first evaluated with linear scan voltammetry (LSV; Figure 4a). All the LSV curves were calibrated with $90 \%$ iR compensation (Figures S19 and S20). The overpotential for achieving a current density of $10 \mathrm{~mA}$ cm-2 is $320 \mathrm{mV}$ for H-CoP@NC, which is higher than that for the commercial benchmark catalyst, RuO2 $(280 \mathrm{mV})$, but lower than that for H-CoP $(360 \mathrm{mV})$ and for CoP/NC (410 mV). Moreover, the Tafel slope of H-CoP@NC is $73 \mathrm{mV}$ dec-1, lower than all the other three tested samples $(79,100$, and 141 $\mathrm{mV}$ dec-1 for RuO2, H-CoP, and CoP/NC, respectively; Figure $4 \mathrm{~b}$ ). The substantial differences in the overpotential and Tafel slope between H-CoP@NC, H-CoP and CoP/NC clearly indicate that H-CoP@NC is capable of delivering much better electrocatalytic activity than H-CoP and CoP/NC. The H-CoP@NC also demonstrates superior durability over $\mathrm{H}-\mathrm{COP}$ and COP/NC, as confirmed by the results from the long-term polarization cycles and constant potential hydrolysis (CPH) tests (Figure 4c,d). The polarization curve of H-CoP@ NC after 1000 cycles shows a negligible change, while that of H-CoP and $\mathrm{CoP} / \mathrm{NC}$ displays noticeable degeneration (Figure 4c). In addition, after $24 \mathrm{~h}$ of testing at a constant overpotential of $320 \mathrm{mV}, \mathrm{H}-\mathrm{CoP} @ \mathrm{NC}$ maintains $95 \%$ of the current density, while H-CoP and CoP/NC only maintain 16 and $45 \%$, respectively.
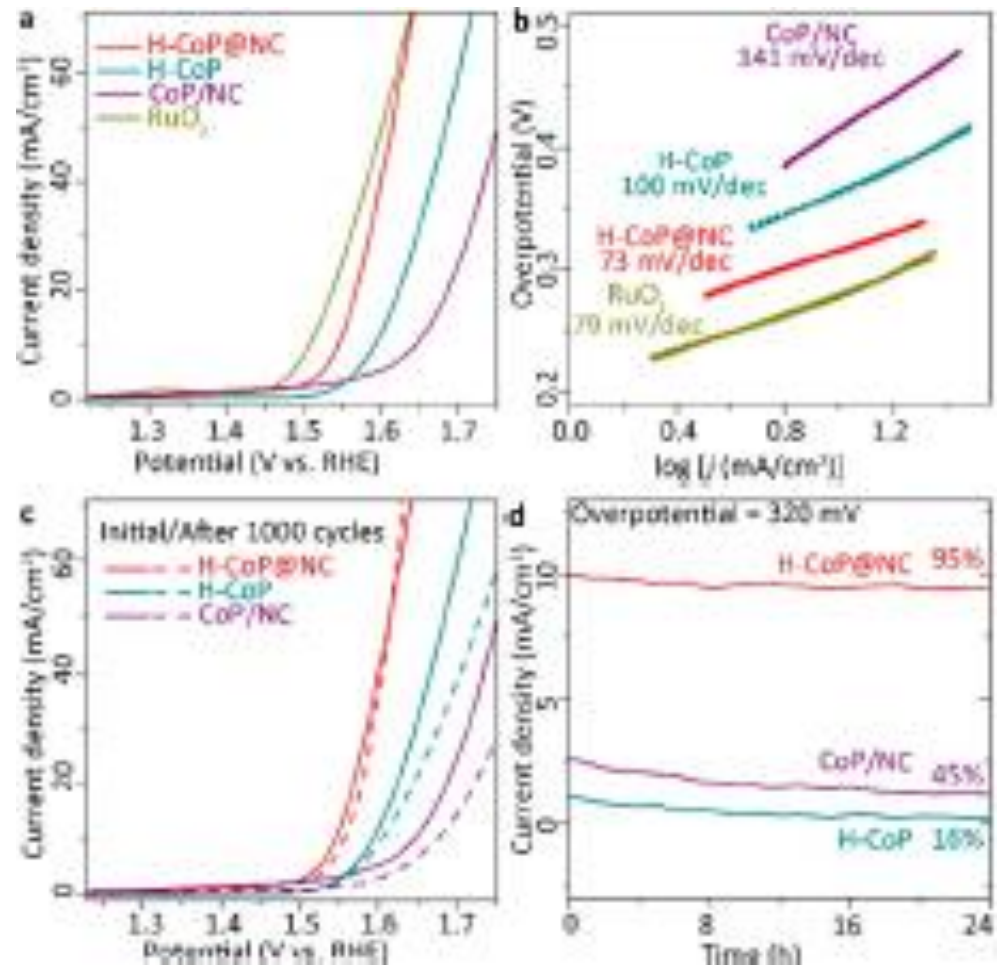

Figure 4. Electrocatalytic activity toward OER of H-COP@NC, H-CoP, CoP/NC evaluated in the solution of $1.0 \mathrm{M} \mathrm{KOH}$ (pH 14). (a) Polarization curves, (b) Tafel plots, (c) polarization curves before and after 1000 cycles, and (d) current density at a static overpotential of $320 \mathrm{mV}$ for $24 \mathrm{~h}(95,16$, and $45 \%)$. The benchmark OER catalyst, RuO2, was also tested for comparison. 

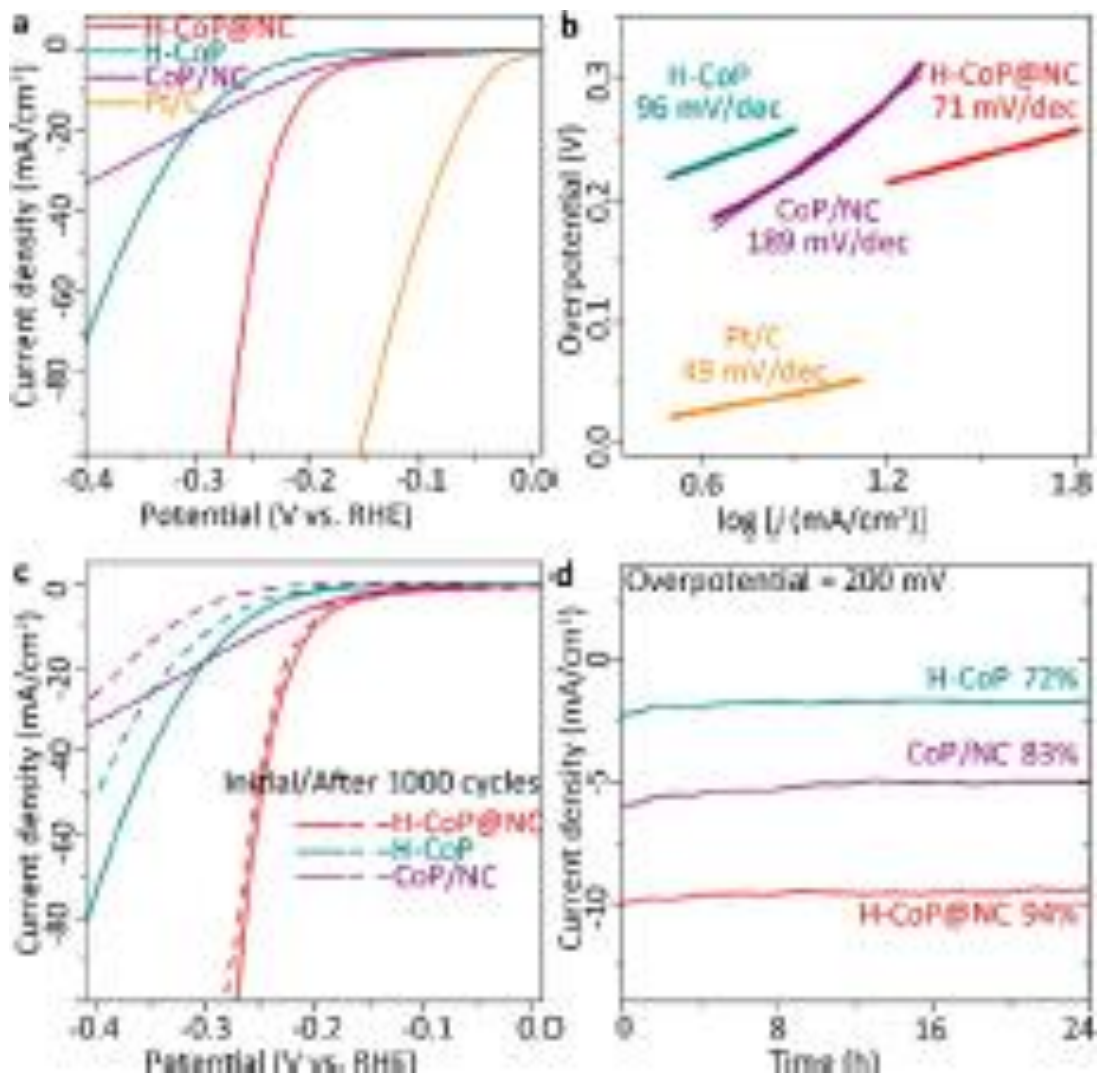

Figure 5. Electrocatalytic activity toward HER of H-CoP@NC, H-CoP, and CoP/NC evaluated in the solution of $1.0 \mathrm{M} \mathrm{KOH}$ (pH 14).(a) Polarization curves, (b) Tafel plots, (c) polarization curves before and after 1000 cycles, and (d) current density at a static overpotential of $200 \mathrm{mV}$ for $24 \mathrm{~h}(94,72$, and $83 \%)$. The benchmark HER catalyst, $\mathrm{Pt} / \mathrm{C}$, was also tested for comparison.

When used as HER electrocatalyst, H-CoP@NC also delivers superior electrocatalytic activity and durability over $\mathrm{H}-\mathrm{CoP}$ and $\mathrm{CoP} / \mathrm{NC}$. With respect to electrocatalytic activity, in the solution of $1.0 \mathrm{M}$ $\mathrm{KOH}$ (pH 14), H-CoP@NC affords an overpotential of $200 \mathrm{mV}$ at a current density of $10 \mathrm{~mA} \mathrm{~cm}-2$ and a Tafel slope of $71 \mathrm{mV}$ dec-1(Figure 5a,b), much smaller than that of H-CoP (269 mV, $96 \mathrm{mV} \mathrm{dec}-1$ ) and CoP/NC (240 mV, $189 \mathrm{mV} \mathrm{dec-1).In} \mathrm{terms} \mathrm{of} \mathrm{durability,} \mathrm{H-CoP@NC} \mathrm{displays} \mathrm{very} \mathrm{slight} \mathrm{variation}$ in the polarization curve after 1000 cycles at a current density of $10 \mathrm{~mA} \mathrm{~cm}-2$ (Figure $5 \mathrm{c}$ ) and retains $94 \%$ of the current density after $24 \mathrm{~h}$ of continuous electrolysis at a static overpotential of $200 \mathrm{mV}$ (Figure $5 \mathrm{~d}$ ). By contrast, $\mathrm{H}-\mathrm{COP}$ and $\mathrm{CoP} / \mathrm{NC}$ exhibit obvious changes in the polarization curve as well as significant reduction in the current density after the long-term polarization cycles and $\mathrm{CPH}$ tests (Figure 5c,d). The great catalytic performances of H-CoP@NC for both OER and HER suggest that HCoP@NC can act as a bifunctional electrocatalysts for OWS. Therefore, a two-electrode system using $\mathrm{Ni}$ foam (NF)-supported H-CoP@ NC as both the cathode for HER and the anode for OER (denoted as $\mathrm{H}-\mathrm{CoP} @ \mathrm{NC|} \mid \mathrm{H}-\mathrm{CoP} @ \mathrm{NC}$ ) in $1.0 \mathrm{M} \mathrm{KOH}$ (pH 14) electrolyte was set up for OWS. For comparison, the NF support and the commercially available benchmark catalysts for OER and HER, that is, RuO2 and $\mathrm{Pt} / \mathrm{C}$, respectively, were also chosen to compose three catalyst couples for OWS, i.e., NF||NF, $\mathrm{Pt} / \mathrm{C}|| \mathrm{Pt} / \mathrm{C}$, and $\mathrm{Pt} / \mathrm{C}|| \mathrm{RuO} 2$, and tested under otherwise identical experimental conditions. As shown in Figure 6a, the cell voltage for H-CoP@NC||H-CoP@NC to reach a current density of $10 \mathrm{~mA} / \mathrm{cm} 2$ is $1.72 \mathrm{~V}$, which is much smaller than that of NF| |NF (1.87 V) and thus indicates that the electrocatalytic activities should be primarily attributed to H-CoP@NC rather than the NF support. The cell voltage for H-CoP@NC||H-CoP@NC is also much lower than that of Pt/C||Pt/C (1.83 V), and even close to that of $\mathrm{Pt} / \mathrm{C}|| \mathrm{RuO} 2(1.67 \mathrm{~V})$, the catalyst couple consisted of the benchmark catalysts for HER and OER. These results clearly demonstrate that the H-CoP@NC is a highly active bifunctional electrocatalyst for OWS. 
In fact, as summarized in Table S1, the electrocatalytic performances of H-CoP@NC are also comparable or even superior to many of the recently developed CoP-based bifunctional electrocatalysts for OWS. In addition to the high activity, the H-CoP@NC||H-CoP@NC based full cell displays excellent durability, as indicated by the experimental observation that (1) the polarization curve shows negligible changes even after 1000 cycles (Figure 6b), (2) the current density does not decay for up to $24 \mathrm{~h}$ at a moderate potential of $1.72 \mathrm{~V}$ (Figure $6 \mathrm{c}$ ), (3) the current density can also remain for over $20 \mathrm{~h}$ at a high potential of $1.88 \mathrm{~V}$ (current density of $50 \mathrm{~mA} \mathrm{~cm}-2$; Figure S21), and (4) the hollow structure is preserved after 1000 cycles LSV test (Figures S22 and S23). The excellent durability of the H-CoP@NC||H-CoP@NC based full cell can be further confirmed by the abundant $\mathrm{O} 2$ and $\mathrm{H} 2$ bubbles generated simultaneously on the H-CoP@NC electrodes even after $24 \mathrm{~h}$ of continuous operation (Figure 6d). To gain insight into the excellent electrocatalytic perform-ances of H-CoP@NC and the large performance differences between H-CoP@NC, H-CoP, and CoP/NC, the kinetics of electrocatalytic OER and HER is analyzed based on the electrochemical surface area (ECSA), BET (Brunauer- Emmett-Teller) surface area and charge transfer resistance. The ESCA of H-CoP@NC, H$\mathrm{CoP}$ and $\mathrm{CoP} / \mathrm{NC}$ is estimated with the electrochemical double-layer capacitance (Cdl), which is calculated from the cyclic voltammetry (CV) curves at different scan rates (Figures S24-S26). HCoP@NC exhibits a much higher Cdl $(2.08 \mathrm{mF} \mathrm{cm}-2)$ than that of $\mathrm{H}-\mathrm{CoP}(0.044 \mathrm{mF} \mathrm{cm}-2)$ and of CoP/NC (0.99 $\mathrm{mF} \mathrm{cm-2)(Figure} \mathrm{S27),} \mathrm{suggesting} \mathrm{that} \mathrm{H-CoP@NC} \mathrm{is} \mathrm{capable} \mathrm{of} \mathrm{exposing} \mathrm{many} \mathrm{more} \mathrm{surface}$ active sites than H-CoP and CoP/NC. Such large ESCA of H-CoP@NC should be attributed to its highly porous, hollow nanostructure. Indeed, based on the N2 adsorption-desorption isotherms (Figure S28), the BET surface area of H-CoP@NC is calculated to be $37 \mathrm{~m} 2 \mathrm{~g}-1$, which is much higher than that of $\mathrm{H}$ CoP (15 m2g-1) and of CoP/NC (11 m2g-1). The much higher ESCA and BET surface area of H-CoP@NC should be the benefit of the carbon skeleton, which prevents the porous nanostructure of H-CoP@NC form collapse and thus avoids the significant decrease in the surface area. Moreover, as shown in the Nyquist plots obtained from electrochemical impedance spectroscopy (EIS) (Figures S29-S31), the HCoP@NC displays a semicircular diameter much smaller than that of $\mathrm{H}-\mathrm{CoP}$ and CoP/NC, thus suggesting much lower charge transfer resistance of H-CoP@NC. The low charge transfer resistance of H-CoP@NC is likely due to its high content of N-doped carbon (22.6 wt. \%; vs 20.6 wt. \% for CoP/NC and 0 for $\mathrm{H}-\mathrm{CoP}$ ) and highly porous hollow nanostructures, which significantly improves the electrical conductivity of H-CoP@ NC and enhance the contact between H-CoP@NC and the electrolyte. Benefiting from the collective contributions from the high ESCA, BET surface area, and electrical conductivity, the H-CoP@NC is capable of affording a much faster catalytic kinetics for HER and OER and thus delivering much better catalytic activity than $\mathrm{H}-\mathrm{CoP}$ and CoP/NC. Moreover, since the carbon skeleton contributes substantially to the high ESCA, BET surface area, and electrical conductivity of the $\mathrm{H}$-CoP@NC, the analysis here actually confirms the critical role of the carbon skeleton in the electrocatalytic activity of H-CoP@NC. In fact, compared with $\mathrm{H}-\mathrm{CoP}$ and $\mathrm{CoP} / \mathrm{NC}$, the superior durability of H-CoP@NC should also be attributed to the N-doped carbon, which effectively stabilizes the small CoP nanoparticles against aggregation and/or collapse during the OER/HER processes to enhance their durability. 

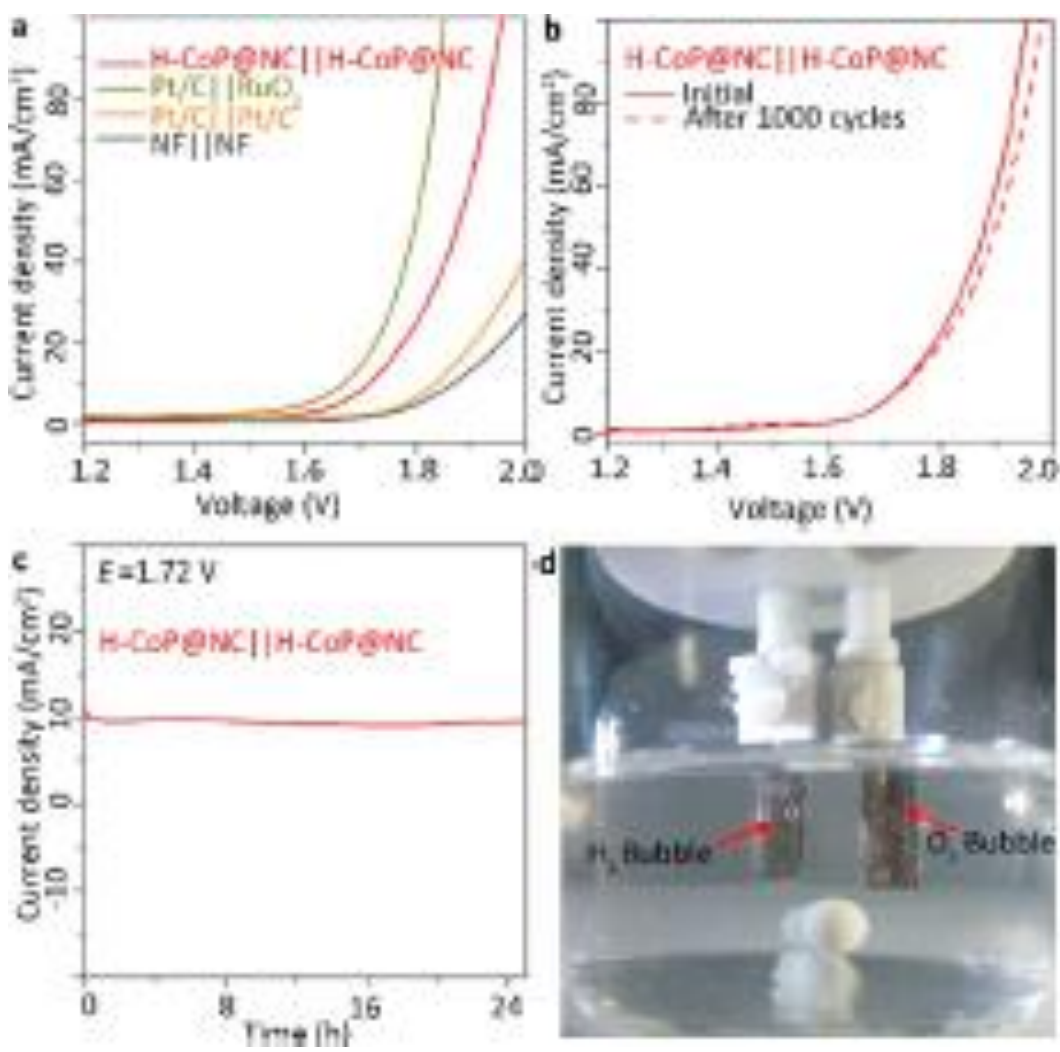

Figure 6. Electrocatalytic activity of H-CoP@NC||H-CoP@NC toward OWS in the solution of $1.0 \mathrm{M} \mathrm{KOH}$ (pH 14). (a) Polarization curves in a two-electrode configuration (catalyst couples of bench-mark catalysts (i.e., $\mathrm{Pt} / \mathrm{C}|| \mathrm{RuO} 2$ and $\mathrm{Pt} / \mathrm{C}|| \mathrm{Pt} / \mathrm{C}$ ) and the electrode support (NF||NF) were also tested for comparison). (b) Polarization curves before and after 1000 cycles. (c) Current density curve at a constant voltage of $1.72 \mathrm{~V}$ for $24 \mathrm{~h}$. (d) Optical photo showing the OWS process.

Conclusion

By coupling the processes of PDA coating and ZIF-67 conversion, we have demonstrated a facile approach for the controlled synthesis of H-CoP@NC nanostructure.H-CoP@ NC features large inner voids and a porous thin shell, which consists of small CoP nanoparticles and conductive $\mathrm{N}$-doped carbon skeleton. Such advantageous nanostructure is of great benefit to the efficient exposure of abundant surface active sites and the significantly enhanced electrical conductivity and stability. Therefore, H-CoP@NC is capable of working as highly active and durable bifunctional electrocatalyst for HER and OER. When further used as the electrocatalyst for OWS, the H-CoP@NC shows great activity close to that of the catalyst couples consisting of $\mathrm{Pt} / \mathrm{C}$ and $\mathrm{RuO} 2$, the benchmark catalysts for HER and OER, respectively. Our work thus provides new insights into the development of TMPs based hollow hybrid nanostructures, particularly those with multiple functionalities in sustainable energy conversion technologies and systems.

References

(1) You, B.; Sun, Y. Innovative Strategies for Electrocatalytic Water Splitting. Acc. Chem. Res. 2018, 51 (7), 1571-1580. 
(2) Li, X.; Hao, X.; Abudula, A.; Guan, G. Nanostructured Catalysts for Electrochemical Water Splitting: Current State and Prospects. J. Mater. Chem. A 2016, 4 (31), 11973-12000.

(3) Hunter, B. M.; Gray, H. B.; Muller, A. M. Earth-Abundant Heterogeneous Water Oxidation Catalysts. Chem. Rev. 2016, 116 (22), 14120-14136.

(4) Lu, F.; Zhou, M.; Zhou, Y.; Zeng, X. First-Row Transition Metal Based Catalysts for the Oxygen Evolution Reaction under Alkaline Conditions: Basic Principles and Recent Advances. Small 2017, 13 (45), 1701931.

(5) Suen, N. T.; Hung, S. F.; Quan, Q.; Zhang, N.; Xu, Y. J.; Chen, H. M. Electrocatalysis for the Oxygen Evolution Reaction: Recent Development and Future Perspectives. Chem. Soc. Rev. 2017, 46 (2), 337-365.

(6) Han, L.; Dong, S.; Wang, E. Transition-Metal (Co, Ni, and Fe)-Based Electrocatalysts for the Water Oxidation Reaction. Adv. Mater. 2016, 28 (42), 9266-9291.

(7) Zeng, M.; Li, Y. Recent Advances in Heterogeneous Electro-catalysts for The Hydrogen Evolution Reaction. J. Mater. Chem. A 2015, 3 (29), 14942-14962.

(8) Jin, H.; Liu, X.; Vasileff, A.; Jiao, Y.; Zhao, Y.; Zheng, Y.; Qiao, S. Z. Single-Crystal Nitrogen-Rich Two-Dimensional Mo5N6 Nano-sheets for Efficient and Stable Seawater Splitting. ACS Nano 2018, 12 (12), 12761-12769.

(9) Zhao, Y.; Jin, B.; Vasileff, A.; Jiao, Y.; Qiao, S.-Z. Interfacial Nickel Nitride/Sulfide as a Bifunctional Electrode for Highly Efficient Overall Water/Seawater Electrolysis. J. Mater. Chem. A 2019, 7 (14), 8117-8121.

(10) Sun, T.; Zhang, S.; Xu, L.; Wang, D.; Li, Y. An Efficient Multifunctional Hybrid Electrocatalyst: Ni2P Nanoparticles on MOF-Derived Co,N-Doped Porous Carbon Polyhedrons for Oxygen Reduction and Water Splitting. Chem. Commun. 2018, 54 (85), 12101-12104.

(11) Popczun, E. J.; McKone, J. R.; Read, C. G.; Biacchi, A. J.; Wiltrout, A. M.; Lewis, N. S.; Schaak, R. E. Nanostructured Nickel Phosphide as an Electrocatalyst for the Hydrogen Evolution Reaction. J. Am. Chem. Soc. 2013, 135 (25), 9267-70.

(12) Popczun, E. J.; Read, C. G.; Roske, C. W.; Lewis, N. S.; Schaak, R. E. Highly Active Electrocatalysis of the Hydrogen Evolution Reaction by Cobalt Phosphide Nanoparticles. Angew. Chem., Int. Ed. 2014, 53 (21), 5427-30.

(13) McEnaney, J. M.; Crompton, J. C.; Callejas, J. F.; Popczun, E. J.; Biacchi, A. J.; Lewis, N. S.; Schaak, R. E. Amorphous Molybdenum Phosphide Nanoparticles for Electrocatalytic Hydrogen Evolution. Chem. Mater. 2014, 26 (16), 4826-4831.

(14) Shi, Y.; Zhang, B. Recent Advances in Transition Metal Phosphide Nanomaterials: Synthesis and Applications in Hydrogen Evolution Reaction. Chem. Soc. Rev. 2016, 45 (6), 1529-41.

(15) Wang, J.; Liu, Z.; Zheng, Y.; Cui, L.; Yang, W.; Liu, J. Recent Advances in Cobalt Phosphide Based Materials for Energy-Related Applications. J. Mater. Chem. A 2017, 5 (44), 22913-22932.

(16) You, B.; Zhang, Y.; Jiao, Y.; Davey, K.; Qiao, S. Z. Negative Charging of Transition-Metal Phosphides via Strong Electronic Coupling for Destabilization of Alkaline Water. Angew. Chem., Int. Ed. 2019, 58 (34), 11796-11800. 
(17) Jin, H.; Liu, X.; Chen, S.; Vasileff, A.; Li, L.; Jiao, Y.; Song, L.; Zheng, Y.; Qiao, S.-Z. HeteroatomDoped Transition Metal Electro-catalysts for Hydrogen Evolution Reaction. ACS Energy Lett. 2019, 4 (4), 805-810.

(18) Yang, X.; Lu, A.-Y.; Zhu, Y.; Hedhili, M. N.; Min, S.; Huang, K.-W.; Han, Y.; Li, L.-J. CoP Nanosheet Assembly Grown on Carbon Cloth: A Highly Efficient Electrocatalyst for Hydrogen Generation. Nano Energy 2015, 15, 634-641.

(19) Liu, Q.; Tian, J.; Cui, W.; Jiang, P.; Cheng, N.; Asiri, A. M.; Sun, X. Carbon Nanotubes Decorated with CoP Nanocrystals: A Highly Active Non-Noble-Metal Nanohybrid Electrocatalyst for Hydrogen Evolution. Angew. Chem., Int. Ed. 2014, 53 (26), 6710-4.

(20) Tian, J.; Chen, J.; Liu, J.; Tian, Q.; Chen, P. Graphene Quantum Dot Engineered Nickel-Cobalt Phosphide as Highly Efficient Bifunctional Catalyst for Overall Water Splitting. Nano Energy 2018, 48, 284-291.

(21) Jiao, L.; Zhou, Y. X.; Jiang, H. L. Metal-Organic Framework-Based CoP/Reduced Graphene Oxide: High-Performance Bifunc-tional Electrocatalyst for Overall Water Splitting. Chem. Sci. 2016, 7 (3), 1690-1695.

(22) Wang, X.; Vasileff, A.; Jiao, Y.; Zheng, Y.; Qiao, S. Z. Electronic and Structural Engineering of Carbon-Based Metal-Free Electro-catalysts for Water Splitting. Adv. Mater. 2019, 31 (13), No. 1803625.

(23) Callejas, J. F.; Read, C. G.; Popczun, E. J.; McEnaney, J. M.; Schaak, R. E. Nanostructured Co2P Electrocatalyst for the Hydrogen Evolution Reaction and Direct Comparison with Morphologically Equivalent CoP. Chem. Mater. 2015, 27 (10), 3769-3774.

(24) Oh, S.; Kim, H.; Kwon, Y.; Kim, M.; Cho, E.; Kwon, H. Porous Co-P Foam as an Efficient Bifunctional Electrocatalyst for Hydrogen And Oxygen Evolution Reactions. J. Mater. Chem. A 2016, 4 (47), 18272

(25) Liu, M.; Li, J. Cobalt Phosphide Hollow Polyhedron as Efficient Bifunctional Electrocatalysts for the Evolution Reaction of Hydrogen and Oxygen. ACS Appl. Mater. Interfaces 2016, 8 (3), 2158-65.

(26) Tan, Y.; Wang, H.; Liu, P.; Cheng, C.; Zhu, F.; Hirata, A.; Chen, M. 3D Nanoporous Metal Phosphides Toward High-Efficiency Electrochemical Hydrogen Production. Adv. Mater. 2016, 28 (15), 2951.

(27) Yu, L.; Hu, H.; Wu, H. B.; Lou, X. W. Complex Hollow Nanostructures: Synthesis and EnergyRelated Applications. Adv. Mater. 2017, 29 (15), 1604563-1604601.

(28) Wei, Y. S.; Zhang, M.; Kitta, M.; Liu, Z.; Horike, S.; Xu, Q. A Single-Crystal Open-Capsule MetalOrganic Framework. J. Am. Chem. Soc. 2019, 141 (19), 7906-7916.

(29) Xie, F.; Zhang, L.; Ye, C.; Jaroniec, M.; Qiao, S. Z. The Application of Hollow Structured Anodes for Sodium-Ion Batteries: From Simple to Complex Systems. Adv. Mater. 2019, 31, No. 1800492.

(30) Pan, Y.; Sun, K.; Liu, S.; Cao, X.; Wu, K.; Cheong, W. C.; Chen, Z.; Wang, Y.; Li, Y.; Liu, Y.; Wang, D.; Peng, Q.; Chen, C.; Li, Y. Core-Shell ZIF-8@ZIF-67-Derived CoP Nanoparticle-Embedded N-Doped Carbon Nanotube Hollow Polyhedron for Efficient Overall Water Splitting. J. Am. Chem. Soc. 2018, 140 (7), 2610-2618. 
(31) Liu, K.; Zhang, C.; Sun, Y.; Zhang, G.; Shen, X.; Zou, F.; Zhang, H.; Wu, Z.; Wegener, E. C.; Taubert, C. J.; Miller, J. T.; Peng, Z.; Zhu, Y. High-Performance Transition Metal Phosphide Alloy Catalyst for Oxygen Evolution Reaction. ACS Nano 2018, 12 (1), 158-167.

(32) Yang, J.; Zhang, F.; Lu, H.; Hong, X.; Jiang, H.; Wu, Y.; Li, Y. Hollow Zn/Co ZIF Particles Derived from Core-Shell ZIF-67@ZIF-8 as Selective Catalyst for the Semi-Hydrogenation of Acetylene. Angew. Chem., Int. Ed. 2015, 54 (37), 10889-93.

(33) Zhou, K.; Mousavi, B.; Luo, Z.; Phatanasri, S.; Chaemchuen, S.; Verpoort, F. Characterization and Properties of Zn/Co Zeolitic Imidazolate Frameworks vs. ZIF-8 and ZIF-67. J. Mater. Chem. A 2017, 5 (3), 952-957.

(34) Wang, S.; Fan, Y.; Teng, J.; Fan, Y. Z.; Jiang, J. J.; Wang, H. P.; Grutzmacher, H.; Wang, D.; Su, C. Y. Nanoreactor Based on Macroporous Single Crystals of Metal-Organic Framework. Small 2016, 12 (41), 5702-5709.

(35) Teng, J.; Chen, M.; Xie, Y.; Wang, D.; Jiang, J.-J.; Li, G.; Wang, H.-P.; Fan, Y.; Wei, Z.-W.; Su, C.Y. Hierarchically Porous Single Nanocrystals of Bimetallic Metal-Organic Framework for Nano-reactors with Enhanced Conversion. Chem. Mater. 2018, 30 (18), 987.

(36) Liang, Z.; Zhao, R.; Qiu, T.; Zou, R.; Xu, Q. Metal-Organic Framework-Derived Materials for Electrochemical Energy Applica-tions. EnergyChem. 2019, 1 (1), 100001.

(37) Hong, W.; Kitta, M.; Xu, Q. Bimetallic MOF-Derived FeCo-P/C Nanocomposites as Efficient Catalysts for Oxygen Evolution Reaction. Small Methods 2018, 2 (12), 1800214.

(38) Wang, S.; Chen, M.; Xie, Y.; Fan, Y.; Wang, D.; Jiang, J. J.; Li, Y.; Grutzmacher, H.; Su, C. Y. Nanoparticle Cookies Derived from Metal-Organic Frameworks: Controlled Synthesis and Application in Anode Materials for Lithium-Ion Batteries. Small 2016, 12 (17), 2365-75.

(39) Wang, S.; Teng, J.; Xie, Y.; Wei, Z.-W.; Fan, Y.; Jiang, J.-J.; Wang, H.-P.; Liu, H.; Wang, D.; Su, C.-Y. Embedding $\mathrm{CoO}$ Nanoparticles in a Yolk-Shell N-doped Porous Carbon Support for Ultrahigh and Stable Lithium Storage. J. Mater. Chem. A 2019, 7 (8), 4036-4046.

(40) Ran, J.; Xiao, L.; Wu, W.; Liu, Y.; Qiu, W.; Wu, J. Zeolitic Imidazolate Framework-8 (ZIF-8) as a Sacrificial Template: One-Pot Synthesis of Hollow Poly(Dopamine) Nanocapsules and Yolk-Structured Poly(Dopamine) Nanocomposites. Nanotechnology 2017, 28 (5), 055604.

(41) Lee, H.; Dellatore, S. M.; Miller, W. M.; Messersmith, P. B. Mussel-Inspired Surface Chemistry for Multifunctional Coatings. Science 2007, 318 (5849), 426-30.

(42) Radhakrishnan, D.; Narayana, C. Guest Dependent Brillouin and Raman Scattering Studies of Zeolitic Imidazolate Framework-8 (ZIF-8) Under External Pressure. J. Chem. Phys. 2016, 144 (13), 134704.

(43) Kumari, G.; Jayaramulu, K.; Maji, T. K.; Narayana, C. Temperature Induced Structural Transformations and Gas Adsorp-tion in the Zeolitic Imidazolate Framework ZIF-8: A Raman Study. J. Phys. Chem. A 2013, 117 (43), 11006-12.

(44) Jiang, Z.; Li, Z.; Qin, Z.; Sun, H.; Jiao, X.; Chen, D. LDH Nanocages Synthesized with MOF Templates and Their High Performance as Supercapacitors. Nanoscale 2013, 5 (23), 11770-5. 
(45) Guo, W.; Liang, Z.; Zhao, J.; Zhu, B.; Cai, K.; Zou, R.; Xu, Q. Hierarchical Cobalt Phosphide Hollow Nanocages toward Electro-catalytic Ammonia Synthesis under Ambient Pressure and Room Temperature. Small Methods 2018, 2 (12), 1800204.

(46) Xu, X.; Zhong, Z.; Yan, X.; Kang, L.; Yao, J. Cobalt Layered Double Hydroxide Nanosheets Synthesized in Water-Methanol Solution as Oxygen Evolution Electrocatalysts. J. Mater. Chem. A 2018, 6 (14), 5999-6006.

(47) Zhao, J.; Xie, Y.; Yuan, W.; Li, D.; Liu, S.; Zheng, B.; Hou, W. A Hierarchical Co-Fe LDH Rope-like Nanostructure: Facile Prepara-tion from Hexagonal Lyotropic Liquid Crystals and Intrinsic Oxidase-like Catalytic Activity. J. Mater. Chem. B 2013, 1 (9), 1263.

(48) Wu, H. B.; Guan, B. Y.; He, P.; Yu, X.-Y. Synthesis of ZIF-67 Nanocubes with Complex Structures Co-Mediated by Dopamine and Polyoxometalate. J. Mater. Chem. A 2018, 6 (40), 19338-19341.

(49) Chung, D. Y.; Jun, S. W.; Yoon, G.; Kim, H.; Yoo, J. M.; Lee, K. S.; Kim, T.; Shin, H.; Sinha, A. K.; Kwon, S. G.; Kang, K.; Hyeon, T.; Sung, Y. E. Large-Scale Synthesis of Carbon-Shell-Coated FeP Nanoparticles for Robust Hydrogen Evolution Reaction Electro-catalyst. J. Am. Chem. Soc. 2017, 139 (19), 6669-6674.

(50) Han, A.; Chen, W.; Zhang, S.; Zhang, M.; Han, Y.; Zhang, J.; Ji, S.; Zheng, L.; Wang, Y.; Gu, L.; Chen, C.; Peng, Q.; Wang, D.; Li, Y. A Polymer Encapsulation Strategy to Synthesize Porous NitrogenDoped Carbon-Nanosphere-Supported Metal Isolated-Single-Atomic-Site Catalysts. Adv. Mater. 2018, 30 (15), No. 1706508.

(51) Liu, R.; Mahurin, S. M.; Li, C.; Unocic, R. R.; Idrobo, J. C.; Gao, H.; Pennycook, S. J.; Dai, S. Dopamine as a Carbon Source: The Controlled Synthesis of Hollow Carbon Spheres and Yolk-Structured Carbon Nanocomposites. Angew. Chem., Int. Ed. 2011, 50 (30), 6799-802.

(52) Postma, A.; Yan, Y.; Wang, Y.; Zelikin, A. N.; Tjipto, E.; Caruso, F. Self-Polymerization of Dopamine as a Versatile and Robust Technique to Prepare Polymer Capsules. Chem. Mater. 2009, 21 (14), 3042-3044.

(53) Cui, Y.-H.; Xue, M.-Z.; Fu, Z.-W.; Wang, X.-L.; Liu, X.-J. Nanocrystalline CoP Thin Film as a New Anode Material for Lithium Ion Battery. J. Alloys Compd. 2013, 555, 283-290.

(54) Li, Y.; Malik, M. A.; O'Brien, P. Synthesis of Single-Crystalline CoP Nanowires by a One-Pot Metal-Organic Route. J. Am. Chem. Soc. 2005, 127 (46), 16020-1.

(55) Li, H.; Yang, P.; Chu, D.; Li, H. Selective Maltose Hydrogenation to Maltitol on a Ternary Co-P-B Amorphous Catalyst and the Synergistic Effects of Alloying B and P. Appl. Catal., A 2007, 325 (1), 34-40.

(56) Jiang, P.; Liu, Q.; Ge, C.; Cui, W.; Pu, Z.; Asiri, A. M.; Sun, X. CoP Nanostructures with Different Morphologies: Synthesis, Characterization and a Study of Their Electrocatalytic Performance Toward the Hydrogen Evolution Reaction. J. Mater. Chem. A 2014, 2 (35), 14634.

(57) Grosvenor, A. P.; Wik, S. D.; Cavell, R. G.; Mar, A. Examination of the Bonding in Binary Transition-Metal Mono-phosphides MP ( $\mathrm{M}=\mathrm{Cr}, \mathrm{Mn}, \mathrm{Fe}, \mathrm{Co})$ by X-Ray Photoelectron Spectroscopy. Inorg. Chem. 2005, 44 (24), 8988-98. 
(58) Burns, A. W.; Layman, K. A.; Bale, D. H.; Bussell, M. E. Understanding the Relationship between Composition and Hydro-desulfurization Properties for Cobalt Phosphide Catalysts. Appl. Catal., A 2008, $343(1-2), 68-76$.

(59) Zheng, F.; Yang, Y.; Chen, Q. High Lithium Anodic Performance of Highly Nitrogen-Doped Porous Carbon Prepared From a Metal-Organic Framework. Nat. Commun. 2014, 5, 5261. 